\title{
Selection Rules for Rotor Transitions in Completely Asymmetric Molecules with Planar Top and Frame and a Twofold Barrier
}

\author{
Thorvald Pedersen and Niels Wessel Larsen \\ Chemical Laboratory V, University of Copenhagen \\ Copenhagen, Denmark \\ and Helmut Dreizler \\ Physikalisches Institut der Universität Freiburg i. Br. * \\ (Z. Naturforsch. 24 a, 649-652 [1969]; received 25 January 1969)
}

\begin{abstract}
In this contribution selection rules for rotation torsion transitions are considered for completely asymmetric molecules. Quade gave two descriptions of this type of molecules resulting in two different invariance groups of both Hamiltonians lead to indentical selection rules.
\end{abstract}

In his paper ${ }^{1}$ QUADE divides the molecules which are planar in their equilibrium position and have planar top and frame into three classes:

Class I: Molecules where top and frame possess two planes of symmetry. The planes intersect in a common $C_{2}$-axis, which is the internal rotation axis. It follows that top and frame are both of $C_{2 v}$-symmetry.

Class II: The top has two planes of symmetry. The frame has only one. The $C_{2}$-axis of the top is the internal rotation axis.

Class III: The frame has two planes of symmetry. The top has one. The $C_{2}$-axis of the frame is now the internal rotation axis.

It is seen that the classification into the last two classes can be chosen at will, the choice being dictated by computational convenience only. Thus the Class II description should be chosen when the part of the molecule with the $C_{2}$-axis has the smaller inertial constants, and vice versa.

The selection rules for the rotortransitions of Class I-molecules have been derived by Dreizler and Möller ${ }^{2}$ and for Class III by Pedersen, LaRSEN and NygaARD ${ }^{3}$. Because a Class III molecule can in principle be described as a Class II case and vice versa, the allowed transitions within the term scheme of a given molecule predicted from the two descriptions should be the same. In the present paper we show that this is the case by developing a

* Neue Anschrift: Institut für Physikalische Chemie der Universität Kiel

1 C. R. Quade, J. Chem. Phys. 47, 1073 [1967].

2 H. Dreizler and K. D. Möller, Z. Naturforsch. 23 a, $1221[1968]$. connection between the class II and the class III description. We proceed along the following lines:

i. The relation between the coordinates used in the two descriptions is derived.

ii. The dipole operators are shown to be identical when expressed in the same coordinates.

iii. The irreducible representations of the invariance groups $G(\mathrm{II})$ and $G(\mathrm{III})$ are correlated by showing that a basis for the subspace of functions carrying $\Gamma_{i}$ of $G(\mathrm{II})$ is related to a basis for a subspace of $\Gamma_{j}$ of $G(\mathrm{III})$ by a unitary transformation, so that the subspaces are identical.

The coordinate systems for Class II and Class III are placed according to the conventions of QUADE and $\operatorname{LIN}^{4}$. The origin is at the center of mass of the

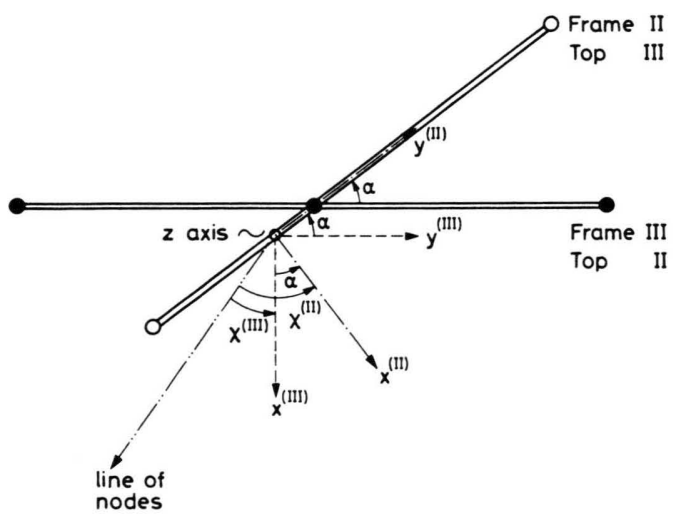

Fig. 1. Coordinate systems used in the Class II and Class III descriptions by $\mathrm{QUADE}^{1}$, viewed from the $z$-axis.

3 Th. Pedersen, N. W. Larsen, and L. Nygaard, Microwave spectra of all the monodeuteriophenols, to be published in J. Mol. Structure.

4 C. R. Quade and C. C. Lin, J. Chem. Phys. 38, 540 [1963]. 
molecule. The $z$-axis is always parallel to the internal rotation axis $\left(C_{2}\right.$-axis). As is seen in Fig. 1 the $y$-axes coincide in the plane of the molecule when $\alpha=0$. For $\alpha \neq 0$ the $y$-axes always remain parallel to the respective frames as described in the Fig. 1. The systems are right-handed.

The angles $\vartheta$ and $\varphi$ are the usual polar angles of the $z$-axis with respect to the space-fixed coordinate system. The third Eulerian angle $\chi$ is measured from the line of nodes to the $x$-axis. It follows that the coordinates used in the two classes differ only with respect to the third Eulerian angle:

$$
\chi^{(\mathrm{II})}=\chi^{(\mathrm{III})}+\alpha .
$$

A sufficient condition for a transition to be allowed is that the space-fixed $Z$-component of the transition moment integral is different from zero:

$$
\int \psi_{v^{\prime}}^{*} \hat{\mu}_{Z} \psi_{v^{\prime \prime}} \mathrm{d} \tau \neq 0
$$

where $v^{\prime}$ and $v^{\prime \prime}$ characterize the eigenfunctions of the Hamiltonian.

For Class II the dipole operator can be written:

$$
\hat{\mu}_{Z}^{(\mathrm{II})}=\hat{\Phi}_{Z y}^{(\mathrm{II})} \mu_{y}+\hat{\Phi}_{Z z}^{(\mathrm{II})} \mu_{z}
$$

where $\mu_{y}$ and $\mu_{z}$ are the components of the dipole moment along the $y$ and $z$ axes in the equilibrium configuration.

$$
\begin{aligned}
& \hat{\Phi}_{Z y}^{(\mathrm{II})}=\cos \chi^{(\mathrm{II})} \sin \vartheta, \\
& \hat{\Phi}_{Z z}^{(\mathrm{II})}=\cos \vartheta .
\end{aligned}
$$

For Class III we get analogously ${ }^{3}$ $\hat{\mu}_{Z}^{(\mathrm{III})}=-\hat{\Phi}_{Z x}^{(\mathrm{III})} \mu_{y} \sin \alpha+\hat{\Phi}_{Z y}^{(\mathrm{III})} \mu_{y} \cos \alpha+\hat{\Phi}_{Z z}^{(\mathrm{III})} \mu_{z}$

where $\mu_{y}$ and $\mu_{z}$ are the same as above.

$$
\begin{aligned}
& \hat{\Phi}_{Z x}^{(\mathrm{III})}=\sin \chi^{(\mathrm{III})} \sin \vartheta, \\
& \hat{\Phi}_{Z y}^{(\mathrm{III})}=\cos \chi^{(\mathrm{III})} \sin \vartheta, \\
& \hat{\Phi}_{Z z}^{(\mathrm{III})}=\cos \vartheta .
\end{aligned}
$$

Inserting (1) in (3) and using (6) and (7) we get:

$$
\hat{\Phi}_{Z y}^{(\mathrm{II})}=-\hat{\Phi}_{Z x}^{(\mathrm{III})} \sin \alpha+\hat{\Phi}_{Z y}^{(\mathrm{III})} \cos \alpha .
$$

By insertion of (8) and (9) in (2) we get (5). So (2) and $(5)$ are equivalent.

It can be shown that the invariance group for the Hamiltonian for Class II is:

$$
G(\mathrm{II})=\left\{E, C_{2}, C_{2 x}^{(\mathrm{II})} \sigma_{v}(y, z), C_{2 x}^{(\mathrm{II})} \sigma_{v}(x, z)\right\}
$$

where

$$
\begin{gathered}
C_{2 x}^{(\mathrm{II})}: \varphi \rightarrow \varphi+\pi ; \vartheta \rightarrow \pi-\vartheta ; \\
\chi^{(\mathrm{II})} \rightarrow \pi-\chi^{(\mathrm{II})} ; \alpha \rightarrow \alpha ;
\end{gathered}
$$

and

$$
\left.\begin{array}{rl}
C_{2}: & \alpha \rightarrow \alpha+\pi \\
\sigma_{v}(y, z): & \alpha \rightarrow-\alpha \\
\sigma_{v}(x, z): & \alpha \rightarrow \pi-\alpha
\end{array}\right\} \begin{aligned}
& \varphi \rightarrow \varphi \\
& \vartheta \rightarrow \vartheta \\
& \chi^{(\mathrm{II})} \rightarrow \chi^{(\mathrm{II})}
\end{aligned}
$$

\begin{tabular}{|c|c|c|c|c|}
\hline $\begin{array}{l}G(\mathrm{II}) \\
G(\mathrm{III})\end{array}$ & $\begin{array}{l}E \\
E\end{array}$ & $\begin{array}{c}C_{2} \\
C_{2 z}^{(\mathrm{III})} C_{2} \\
\end{array}$ & $\begin{array}{c}C_{2 x}^{(\mathrm{II})} \sigma_{v}(y, z) \\
C_{2 x}^{(\mathrm{III})} \sigma_{v}(y, z)\end{array}$ & $\begin{array}{l}C_{2 x}^{(\mathrm{II})} \sigma_{v}(x, z) \\
C_{2 y}^{(\mathrm{III})} \sigma_{v}(x, z)\end{array}$ \\
\hline $\mathrm{A}_{1}$ & 1 & 1 & 1 & 1 \\
\hline $\mathrm{A}_{2}$ & 1 & 1 & -1 & -1 \\
\hline $\mathrm{B}_{1}$ & 1 & -1 & 1 & -1 \\
\hline $\mathrm{B}_{2}$ & 1 & -1 & -1 & 1 \\
\hline
\end{tabular}

and for Class III the group is 3 :

$G(\mathrm{III})=\left\{E, C_{2 z}^{(\mathrm{III})} C_{2}, C_{2 x}^{(\mathrm{III})} \sigma_{v}(y, z), C_{2 y}^{(\mathrm{III})} \sigma_{v}(x, z)\right\}$

where

$$
\begin{aligned}
& C_{2 x}^{(\mathrm{III})}: \varphi \rightarrow \varphi+\pi ; \vartheta \rightarrow \pi-\vartheta ; \chi^{(\mathrm{III})} \rightarrow \pi-\chi^{(\mathrm{III})} ; \\
& \alpha \rightarrow \alpha \text {; } \\
& C_{2 y}^{(\mathrm{III})}: \varphi \rightarrow \varphi+\pi ; \vartheta \rightarrow \pi-\vartheta ; \chi^{(\mathrm{III})} \rightarrow-\chi^{(\mathrm{III})} \text {; } \\
& \alpha \rightarrow \alpha \text {; }
\end{aligned}
$$

$C_{2 z}^{(\mathrm{III})}: \varphi \rightarrow \varphi ; \vartheta \rightarrow \vartheta ; \chi^{(\mathrm{III})} \rightarrow \chi^{(\mathrm{III})}+\pi ; \alpha \rightarrow \alpha ;$

$G(\mathrm{II})$ and $G(\mathrm{III})$ are both isomorphic with $C_{2 v}$.

We associate the elements $C_{2}$ and $C_{2 z} C_{2}$ with the $C_{2}$ operation of the usual point group, since they are rotations with respect to $\alpha$. We therefore conform with the common use of $\mathrm{A}$ and $\mathrm{B}$ representations. However we do not conform with ${ }^{2}$ in this respect.

The irreducible representations have been chosen in Table 1.

Table 1. Irreducible representations of $G(\mathrm{II})$ and $G(\mathrm{III})$.

We now consider the following bases for the two classes:

$$
\begin{gathered}
\psi^{(\mathrm{II})}=S_{J|K| \gamma M}^{(\mathrm{II})} \varphi_{n \sigma}^{(\mathrm{II})} \\
\psi^{(\mathrm{III})}=S_{J|K| \gamma M}^{(\mathrm{III})} \varphi_{n \sigma}^{(\mathrm{III})}
\end{gathered}
$$

where $S_{J|K| \gamma M}^{(\mathrm{II})}$ and $S_{J|K| \gamma M}^{(\mathrm{III})}$ are the Wang functions ${ }^{5}$ in the respective coordinates. $\varphi_{n \sigma}$ are the Mathieufunctions ${ }^{1,6}$ :

$$
\begin{array}{ll}
\varphi n A_{1}=\sum_{k} \operatorname{De}_{2 k}^{(2 r)}(-1)^{k} \cos 2 k a & n=2 r, \\
\varphi n B_{1}=\sum_{k} \operatorname{Do}_{2 k+1}^{(2 r+1)}(-1)^{k} \cos (2 k+1) \alpha & n=2 r, \\
\varphi n A_{2}=\sum_{k} \operatorname{Do}_{(2 k)}^{(2 r)}(-1)^{k} \sin 2 k \alpha & n=2 r+1, \\
\varphi n B_{2}=\sum_{k} \operatorname{De}_{2 k+1}^{(2 r+1)}(-1)^{k+1} \sin (2 k+1) \alpha & n=2 r+1 .
\end{array}
$$

5 H. Dreizler, Fortschr. Chem. Forsch. 10, 59 [1968]. 
Tables 2 and 3 contain the classifications of the functions and the dipole operators under the irreducible representations of the groups $G(\mathrm{II})$ and $G(\mathrm{III})$. One observes that $\varphi_{n \sigma}^{(\mathrm{II})}$ and $\varphi_{n \sigma}^{(\mathrm{III})}$ with the same $n, \sigma$ belong to species with the same label in $G$ (II) and $G($ III).

We now proceed to prove the statements of point iii: The Wang functions are for Class II:

$$
\begin{array}{rlrl}
S_{J|K| \gamma M}^{(\mathrm{II})} & =\left(\psi_{J K M}^{\times(\mathrm{II})}+(-1)^{\gamma} \psi_{J-K M}^{\times(\mathrm{II})}\right) / \sqrt{2}, K & \neq 0 \\
S_{J 00 M}^{(\mathrm{II})} & =\psi_{J O M}^{\mathrm{x}(\mathrm{II})}, & K & =0
\end{array}
$$

where

$$
\psi_{J K M}^{\times(\mathrm{II})}=(-1)^{\max K, M} \Theta_{(\theta)}^{J K M}(\vartheta) e^{i M \varphi} e^{i K \chi}
$$

and analogously for Class III.

By insertion of (1) we get:

$$
\psi_{J K M}^{\mathrm{x}(\mathrm{II})}=\psi_{J K M}^{\mathrm{x}(\mathrm{III})} e^{i K \alpha} .
$$

For $\psi_{J K M}^{\times(\mathrm{III})}$ the following transformation properties hold:

$$
\begin{aligned}
& C_{2 x}^{(\mathrm{III})} \psi_{J K M}^{\times(\mathrm{III})}=(-1)^{J+K} \psi_{J-K M}^{\times(\mathrm{III})}, \\
& C_{2 y}^{(\mathrm{III})} \psi_{J K M}^{\times(\mathrm{III})}=(-1)^{J} \psi_{J-K M}^{\times(\mathrm{III})}, \\
& C_{2 z}^{(\mathrm{III})} \psi_{J K M}^{\times(\mathrm{III})}=(-\mathrm{I})^{K} \psi_{J-K M}^{\times(\mathrm{III})} .
\end{aligned}
$$

In consequence the following transformations hold for $\psi_{J K M}^{\mathrm{x}(\mathrm{II})}$ under the operations of $G(\mathrm{III})$ :

$$
\begin{gathered}
C_{2 z}^{(\mathrm{III})} C_{2} \psi_{J K M}^{\times(\mathrm{II})}=C_{2 z}^{(\mathrm{III})} \psi_{J K M}^{\times(\mathrm{III})} C_{2} e^{i K \alpha}=\psi_{J K M}^{\times(\mathrm{II}),} \\
C_{2 x}^{(\mathrm{III})} \sigma_{v}(y, z) \psi_{J K M}^{\times(\mathrm{II})}=C_{2 x}^{(\mathrm{III})} \psi_{J K M}^{\times(\mathrm{III})} \sigma_{v}(y, z) e^{i K \alpha} \\
=(-1)^{J+K} \psi_{J-K M}^{\mathrm{x}(\mathrm{II})}, \quad(17) \\
C_{2 y}^{(\mathrm{II})} \sigma_{v}(x, z) \psi_{J K M}^{\times(\mathrm{II})}=C_{2 y}^{(\mathrm{III})} \psi_{J K M}^{\times(\mathrm{III})} \sigma_{v}(x, z) e^{i K \alpha} \\
=(-1)^{J+K} \psi_{J-K M}^{\times(\mathrm{II})} .
\end{gathered}
$$

By applying the operations of $G$ (III) on the Wang functions $S_{J|K| \gamma M}^{(\mathrm{II})}$ it is therefore found that these functions, which are either of $A_{1}$ or $A_{2}$ species in $G$ (II) will be of the species with the same label in $G$ (III). It should be noted that these are not equal to the Wang functions of Class III. Therefore, the set of product functions of a given species in $G(\mathrm{II})$ makes up a unitary basis for all functions of that species in $G$ (II) as well as in $G$ (III). So the functionspaces labelled by the same letter are identical. Obviously the product-functions of Table 3 make up another unitary basis for the same space. This basis should therefore be related to the former by a unitary transformation.

6 Tables relating to Mathieu functions, Columbia University Press, New York 1951.
The matrix connecting the two bases can be determined in the following way: the basis $\psi^{\times}(\mathrm{II})$ (A row-matrix containing all the functions $\Psi_{J K M}^{\times}$) can be written as:

$$
\boldsymbol{\psi} \times(\mathrm{II})=\hat{R}_{z}(\alpha) \boldsymbol{\psi}^{\times}(\mathrm{III})=\boldsymbol{\psi}^{\times}(\mathrm{III}) \boldsymbol{R}_{z}(\alpha)
$$

where $\hat{R_{z}}(\alpha)$ is the rotation operator:

$$
\hat{R}_{z}(\alpha)=\exp \left(i \alpha \hat{P}_{z}\right)=\hat{1}+i \alpha \hat{P}_{z}-\alpha^{2} / 2 \hat{P}_{z}^{2} \ldots
$$

where

$$
\hat{P}_{z}=-i \partial / \partial \chi^{(\mathrm{III})} \text {. }
$$

The matrix of $\hat{R}_{z}(\alpha)$ is a diagonal matrix since $\psi_{J K M}^{\mathrm{x}}$ is an eigenfunction of $P_{z}^{n}(n=0,1,2, \ldots)$ with the eigenvalues $K^{n}$. The matrix elements of $\boldsymbol{R}_{z}$ are therefore simply: $\exp (i K \alpha)$ as was also found above (15).

The Wang bases are therefore also related since:

$$
\begin{aligned}
& \boldsymbol{S}(\mathrm{II})=\boldsymbol{\psi} \times(\mathrm{II}) \boldsymbol{W}, \\
& \boldsymbol{S}(\mathrm{III})=\boldsymbol{\psi} \times(\mathrm{III}) \boldsymbol{W}
\end{aligned}
$$

where the matrixelements of $\boldsymbol{W}$ follow from (13). Thus from (18), (19), (20) we get

$\boldsymbol{S}(\mathrm{II})=\boldsymbol{\psi}^{\times}(\mathrm{III}) \boldsymbol{R}_{z}(\alpha) \boldsymbol{W}=\boldsymbol{S}(\mathrm{III}) \boldsymbol{W}^{+} \boldsymbol{R}_{z}(\alpha) \boldsymbol{W}$.

Now (10) and (11) can be written in matrix notation as the direct products of the Wang bases with the Mathieu bases:

$$
\begin{aligned}
& \Psi(\mathrm{II})=\boldsymbol{S}(\mathrm{II}) \otimes \boldsymbol{\varphi}(\mathrm{II}), \\
& \Psi(\mathrm{III})=\boldsymbol{S}(\mathrm{III}) \otimes \boldsymbol{\varphi}(\mathrm{III}) .
\end{aligned}
$$

We therefore need a relation between the Mathieu bases, which are different because the torsional equation is different for the two classes. But since both bases are related to the Fourier basis by matrices $\boldsymbol{D}$ (II) and $\boldsymbol{D}$ (III) [cf. (12)] the two bases are related by:

$$
\boldsymbol{\varphi}(\mathrm{II})=\boldsymbol{\varphi}(\mathrm{III})(\boldsymbol{D}(\mathrm{III}))^{+} \boldsymbol{D}(\mathrm{II}) .
$$

From (22), (23), and (24) we get:

$\Psi(\mathrm{II})=\boldsymbol{S}(\mathrm{III}) \boldsymbol{W}^{+} \boldsymbol{R}_{z}(\alpha) \boldsymbol{W} \boldsymbol{\varphi}(\mathrm{III})(\boldsymbol{D}(\mathrm{III}))^{+} \boldsymbol{D}(\mathrm{II})$

But the direct product in Eq. (25) can be written as : $\Psi(\mathrm{II})=\boldsymbol{S}(\mathrm{III}) \otimes \boldsymbol{\varphi}(\mathrm{III}) \boldsymbol{W}^{+} \boldsymbol{R}_{z}(\alpha) \boldsymbol{W} \otimes(\boldsymbol{D}(\mathrm{III}))^{+} \boldsymbol{D}(\mathrm{II})$

so that the relation of the two bases is now established:

$$
\Psi(\mathrm{II})=\Psi(\mathrm{III}) \boldsymbol{U}
$$




\begin{tabular}{|c|c|c|c|}
\hline \multicolumn{2}{|c|}{ Function or operator } & $J+K+\gamma$ & Species \\
\hline \multirow{2}{*}{\multicolumn{2}{|c|}{$S_{J \mid K \gamma M}^{(\mathrm{II})}$}} & $\mathrm{e}$ & $\mathrm{A}_{1}$ \\
\hline & & o & $\mathrm{A}_{2}$ \\
\hline \multicolumn{2}{|l|}{$\varphi n A_{1}$} & & $\mathrm{~A}_{1}$ \\
\hline \multicolumn{2}{|l|}{$\varphi n B_{1}$} & & $\mathrm{~B}_{1}$ \\
\hline \multicolumn{2}{|l|}{$\varphi n A_{2}$} & & $\mathrm{~A}_{2}$ \\
\hline \multicolumn{2}{|l|}{$\varphi n B_{2}$} & & $\mathrm{~B}_{2}$ \\
\hline \multirow[t]{8}{*}{$S_{J K \gamma M}^{(\mathrm{II})}$} & $\cdot q n A_{1}$ & $\mathrm{e}$ & $\mathrm{A}_{1}$ \\
\hline & $\varphi n A_{2}$ & o & $\mathrm{A}_{1}$ \\
\hline & $\varphi n A_{1}$ & o & $\mathrm{A}_{2}$ \\
\hline & $\varphi n A_{2}$ & e & $\mathrm{A}_{2}$ \\
\hline & on $B_{1}$ & e & $\mathrm{B}_{1}$ \\
\hline & $\varphi n B_{2}$ & o & $\mathrm{B}_{1}$ \\
\hline & $\varphi n B_{1}$ & o & $\mathrm{B}_{2}$ \\
\hline & $\varphi n B_{2}$ & $\mathrm{e}$ & $\mathrm{B}_{2}$ \\
\hline & & $\mathrm{A}_{2}$ \\
\hline \multirow{2}{*}{\multicolumn{2}{|c|}{$\hat{\Phi}_{\mathrm{zz}}^{(\mathrm{II})}$}} & & $\mathrm{A}_{2}$ \\
\hline$\hat{\mu} z$ & & & $\mathrm{~A}_{2}$ \\
\hline
\end{tabular}

\begin{tabular}{|c|c|c|c|c|}
\hline \multicolumn{2}{|c|}{ Function or operator } & $K$ & $J+\gamma$ & Species \\
\hline \multirow{4}{*}{\multicolumn{2}{|c|}{$S_{J|K| \gamma M}^{(\mathrm{IIII})}$}} & $\mathrm{e}$ & $\mathrm{e}$ & $A_{1}$ \\
\hline & & e & o & $\mathrm{A}_{2}$ \\
\hline & & o & o & $\mathrm{B}_{1}$ \\
\hline & & o & e & $\mathrm{B}_{2}$ \\
\hline \multicolumn{2}{|l|}{$\varphi n A_{1}$} & & & $A_{1}$ \\
\hline \multicolumn{2}{|l|}{$\varphi n B_{1}$} & & & $\mathrm{~B}_{1}$ \\
\hline \multicolumn{2}{|l|}{$\varphi n A_{2}$} & & & $\mathrm{~A}_{2}$ \\
\hline \multicolumn{2}{|l|}{$\varphi n B_{2}$} & & & $\mathrm{~B}_{2}$ \\
\hline \multirow[t]{16}{*}{$S_{J|K| \gamma M}^{(I I I)}$} & $\cdot \varphi n A_{1}$ & e & $\mathrm{e}$ & $\mathrm{A}_{1}$ \\
\hline & $\varphi n A_{2}$ & e & o & $\mathrm{A}_{1}$ \\
\hline & $\varphi n B_{1}$ & 0 & o & $\mathrm{A}_{1}$ \\
\hline & $\varphi n B_{2}$ & o & $\mathrm{e}$ & $A_{1}$ \\
\hline & $\varphi n A_{2}$ & e & e & $\mathrm{A}_{2}$ \\
\hline & $\varphi n A_{1}$ & e & o & $\mathrm{A}_{2}$ \\
\hline & $\varphi n B_{2}$ & o & o & $\mathrm{A}_{2}$ \\
\hline & $\varphi n B_{1}$ & o & $\mathrm{e}$ & $\mathrm{A}_{2}$ \\
\hline & $\varphi n B_{1}$ & e & e & $\mathrm{B}_{1}$ \\
\hline & $\varphi \cap B_{2}$ & e & o & $\mathrm{B}_{1}$ \\
\hline & $\varphi n A_{1}$ & o & o & $\mathrm{B}_{1}$ \\
\hline & $\varphi n A_{2}$ & o & e & $\mathrm{B}_{1}$ \\
\hline & $\varphi n B_{2}$ & e & $\mathrm{e}$ & $\mathrm{B}_{2}$ \\
\hline & $\varphi n B_{1}$ & e & o & $\mathrm{B}_{2}$ \\
\hline & $\varphi n A_{2}$ & o & o & $\mathrm{B}_{2}$ \\
\hline & $\varphi n A_{1}$ & o & e & $\mathrm{B}_{2}$ \\
\hline \multicolumn{2}{|c|}{$\widehat{\Phi}_{z z}^{(I I I)} \sin \alpha$} & & & $\mathrm{A}_{2}$ \\
\hline \multicolumn{2}{|c|}{$\widehat{\Phi}_{z y}^{(\text {III })} \cos \alpha$} & & & $\mathrm{A}_{2}$ \\
\hline \multicolumn{2}{|c|}{$\widehat{\Phi}_{z x}^{(\text {III })}$} & & & $\mathrm{A}_{2}$ \\
\hline \multicolumn{2}{|l|}{$\hat{\mu}_{Z}$} & & & $\mathrm{~A}_{2}$ \\
\hline
\end{tabular}

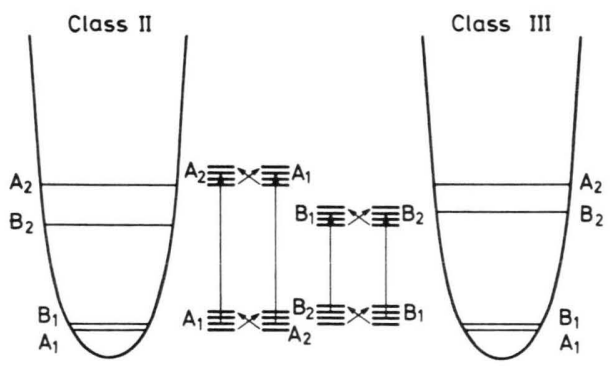
Table 3. Functions and operators carrying irreducible representations of $G(\mathrm{III})$.

where $\quad \boldsymbol{U}=\boldsymbol{W}^{+} \boldsymbol{R}_{z}(\alpha) \boldsymbol{W} \otimes(\boldsymbol{D}(\mathrm{III}))^{+} \boldsymbol{D}(\mathrm{II})$.

Fig. 2. Schematic term scheme of one molecule in the Class II and Class III descriptions. The pure torsional levels are given on both sides. They are different in the two cases. The rotorsional levels and representative transitions are given in the middle. Observe that e.g. an $\mathrm{A}_{1}$ rotorsional eigenfunction gets contributions only from productfunctions which contain A torsional functions in the Class II case. But in the Class III case the same rotorsional eigenfunctions gets contributions from productfunctions which contain $\mathrm{A}$ as well as $\mathrm{B}$ torsional functions.

It remains to show that the selection rules are identical but that follows from the Tables 2 and 3 since $\mu_{Z}$ has the same species in the two groups $\left(\mathrm{A}_{2}\right)$.

We therefore get:

$$
\mathrm{A}_{1} \leftrightarrow \mathrm{A}_{2}, \quad \mathrm{~B}_{1} \leftrightarrow \mathrm{B}_{2}
$$

for both classes as illustrated in Fig. 2. The selection rules on $J$ are the usual ones: $\Delta J=0, \pm 1$. 